\title{
Global existence of strong solutions to the micro- polar, compressible flow with density-dependent viscosities
}

\author{
Mingtao Chen ${ }^{1,2}$
}

Correspondence: mtchen@sdu.edu. $\mathrm{cn}$

${ }^{1}$ College of Mathematical Sciences, Xiamen University, Xiamen 361005, PR China

Full list of author information is available at the end of the article

\section{Abstract}

This article is concerned with global strong solutions of the micro-polar, compressible flow with density-dependent viscosity coefficients in one-dimensional bounded intervals. The important point in this article is that the initial density may vanish in an open subset.

\section{Introduction}

Theory of micro-polar, compressible flow was first introduced by Eringen [1], describing the compressible fluids with randomly oriented particles suspended in the medium when the deformation of fluid particles is ignored. The governing equations in Eulerian coordinate take the form as

$$
\left\{\begin{array}{l}
\rho_{t}+(\rho u)_{x}=0 \\
(\rho u)_{t}+\left(\rho u^{2}\right)_{x}+p_{x}=\left(\mu u_{x}\right)_{x} \\
(\rho w)_{t}+(\rho u w)_{x}+2 v w=\left(\lambda w_{x}\right)_{x} \\
(\rho e)_{t}+(\rho e u)_{x}+p u_{x}=\mu\left(u_{x}\right)^{2}+\lambda\left(w_{x}\right)^{2}+2 \mu w^{2}+\left(\kappa \theta_{x}\right)_{x}
\end{array}\right.
$$

where $\rho=\rho(t, x)$ denotes the density of the fluid, $u=u(t, x)$ is the velocity, $w=w(t, x)$ is the micro-rotational velocity, $\theta=\theta(t, x)$ is the temperature, $e=e(t, x)$ is the internal energy, $p=p(\rho, \theta)$ is the pressure. $\mu=\mu(\rho, \theta), v=v(\rho, \theta)$, and $\lambda=\lambda(\rho, \theta)$ are the viscosities of the fluid, and $\kappa$ is the heat conductivity.

There are several articles that have considered the above micro-polar, compressible flow, with the viscosity being constant satisfying some physical meaning. Here, we only refer the reader to [2-4], wherein the global existence was established for (1), with the condition that the initial density needs to be bounded a way from zero.

In view of their being physically important, the viscosities are not constants. In this article, we consider a simpler model (2) below. For the physical consideration, in the case of isothermal flow, [5] introduce the viscosities depending on the density $\rho$ for isentropic flow. For the micro-polar, compressible flow, the model meets the following conditions: 


$$
\left\{\begin{array}{l}
\rho_{t}+(\rho u)_{x}=0 \\
(\rho u)_{t}+\left(\rho u^{2}\right)_{x}+(p(\rho))_{x}=\left(\mu(\rho) u_{x}\right)_{x} \\
(\rho w)_{t}+(\rho u w)_{x}+v(\rho) w=\left(\lambda(\rho) w_{x}\right)_{x}
\end{array}\right.
$$

with the initial and boundary conditions:

$$
\left\{\begin{array}{l}
\rho(0, x)=\rho_{0}(x), \quad u(0, x)=u_{0}(x), \quad w(0, x)=w_{0}(x) \quad \text { on } 0 \leq x \leq 1, \\
u(t, 0)=u(t, 1)=w(t, 0)=w(t, 1)=0 .
\end{array}\right.
$$

The pressure $p$ is determined by $p(\rho)=a \rho^{\gamma}$, where $a$ is some positive constant and $\gamma>1$, and we normalize $a=1$ in the rest of this article. The viscosities tend to depend on the density $\rho$, i.e. $\mu(\rho), v(\rho)$, and $\lambda(\rho)$, satisfying

$$
\left\{\begin{array}{l}
\mu(\rho), \quad v(\rho), \quad \lambda(\rho) \in C^{1}[0, \infty) \text { and } \quad \mu(\rho) \geq \mu_{1}>0 \\
\lambda(\rho) \geq \lambda_{1}>0 \quad \text { for all } \rho \geq 0
\end{array}\right.
$$

where $\mu_{1}$ and $\lambda_{1}$ are positive constants.

Our main concern here is to show the existence of global strong solution for the initial boundary value problem (2)-(3). It is worth emphasizing that the initial density may vanish in an open subset, and the viscosity coefficients $\mu, v$, and $\lambda$ depend on density $\rho$.

Some of the relevant studies in this direction can be summarized as follows. When the viscosity $\mu, v$, and $\lambda$ are constants, the global strong solution is established by Chen in [6] where the vacuum is also allowed. We also refer the reader for a detailed description of three-dimensional micro-polar, compressible flow under the effect of magnetic field, in respect of which global weak solution was established by Amirat and Hamdache in [7].

Without the randomly oriented particles suspended in the fluid, i.e., when $w=0$, the compressible Navier-Stokes equation with density-dependent viscosity, Wen and Yao [8] proved the global strong solution in one dimension, which generalized Hoff's study [9] (dealing with the case of constant viscosity coefficient); for the free boundary, the existence of global weak solutions, we refer the readers to Guo and Zhu [10], and Jiang, Xin and Zhang [11] and references therein.

The aim of this article is to consider the micro-polar, compressible flow with density-dependent viscosities, in the spirit of [8].

Now, we state our main result:

Theorem 1.1. Assume that the viscosity $\mu(\rho), v(\rho)$, and $\lambda(\rho)$ satisfy (4), with the initial data $\rho_{0} \in H^{1}(0,1),\left(u_{0}, w_{0}\right) \in H_{0}^{1}(0,1)$. Then, there exists a global strong solutions $(\rho, u, w)$ to the initial boundary value problem (2)-(3) such that for all $T \in(0,+\infty)$,

$$
\left\{\begin{array}{l}
\rho \in L^{\infty}\left(0, T ; H^{1}(0,1)\right), \quad(u, w) \in L^{\infty}\left(0, T ; H_{0}^{1}(0,1)\right), \\
\rho_{t} \in L^{\infty}\left(0, T ; L^{2}(0,1)\right), \quad\left((\rho u)_{t^{\prime}}(\rho w)_{t}\right) \in L^{2}\left(0, T ; L^{2}(0,1)\right), \\
\left(u_{x x}, w_{x x}\right) \in L^{2}\left(0, T ; L^{2}(0,1)\right) .
\end{array}\right.
$$

This article is organized as follows. In Section 2, we derive some uniform estimates for the proof of the main Theorem 1.1, which do not depend on the lower bound of the density. We shall complete the proof of Theorem 1.1 in Section 3.

Notations Throughout this article, we denote $C$, a generic positive constant, depending only on $\rho_{0}, u_{0}, w_{0}$, and the time $T$, but independent of lower bounds of the initial 
density; we will also use the following simplified notations for the standard Sobolev spaces:

$$
L^{p}=L^{p}(0,1), \quad H^{1}=H^{1}(0,1), \quad H_{0}^{1}=H_{0}^{1}(0,1) \quad \text { for some } 1 \leq p \leq \infty .
$$

\section{Uniform estimates}

The following lemma provides standard (energy) estimates which can be obtained by multiplying $(2)_{2}$ by $u$ and $(2)_{3}$ by $w$, and then integrating over $(0, T) \times(0,1)$, with the help of $(2)_{1}$.

Lemma 2.1. Under the conditions of Theorem 1.1, we have

$$
\begin{aligned}
& \sup _{0 \leq t \leq T}\left(|\sqrt{\rho} u|_{L^{2}}^{2}+|p(\rho)|_{L^{1}}\right)+\int_{0}^{T} \int_{0}^{1} \mu(\rho) u_{x}^{2} \mathrm{~d} x \mathrm{~d} t \leq C ; \\
& \sup _{0 \leq t \leq T}|\sqrt{\rho} w|_{L^{2}}^{2}+\int_{0}^{T} \int_{0}^{1} v(\rho) w^{2}+\lambda(\rho) w_{x}^{2} \mathrm{~d} x \mathrm{~d} t \leq C .
\end{aligned}
$$

The following lemma 2.2 is proved in [8], we omit it here, which plays crucial role for the proof of Theorem 1.1.

Lemma 2.2. Under the conditions of Theorem 1.1, we have

$$
\sup _{0 \leq t \leq T}|\rho(t)|_{L^{\infty}} \leq C .
$$

Now we will prove the second crucial estimates.

Lemma 2.3. Under the conditions of Theorem 1.1, we have

$$
\begin{aligned}
& \sup _{0 \leq t \leq T}|u|_{H^{1}}+\int_{0}^{T}\left|\sqrt{\rho} u_{t}\right|_{L^{2}}^{2} \mathrm{~d} t \leq C, \\
& \sup _{0 \leq t \leq T}|w|_{H^{1}}+\int_{0}^{T}\left|\sqrt{\rho} w_{t}\right|_{L^{2}}^{2} \mathrm{~d} t \leq C .
\end{aligned}
$$

Proof. Equation (9) can be obtained via [8], and so we focus on the proof of (10). From $(2)_{3}$, we have

$$
\rho w_{t}+\rho u w_{x}+v(\rho) w=\left(\lambda(\rho) w_{x}\right)_{x} .
$$

Multiplying the above equality by $w_{t}$, integrating the resultant equality with respect to $x$ over $[0,1]$, with the help of Young's inequality and $(2)_{1}$, one gets

$$
\begin{aligned}
& \int_{0}^{1} \rho w_{t}^{2} \mathrm{~d} x+\frac{\mathrm{d}}{\mathrm{d} t} \int_{0}^{1} v(\rho) w^{2}+\lambda(\rho) w_{x}^{2} \mathrm{~d} x \\
& \leq \int_{0}^{1} \rho u^{2} w_{x}^{2} \mathrm{~d} x-\int_{0}^{1}(v(\rho))_{x} u w^{2} \mathrm{~d} x-\int_{0}^{1}(\lambda(\rho))_{x} u w_{x}^{2} \mathrm{~d} x \\
& \quad-\int_{0}^{1} v^{\prime}(\rho) \rho u_{x} w^{2} \mathrm{~d} x-\int_{0}^{1} \lambda^{\prime}(\rho) \rho u_{x} w_{x}^{2} \mathrm{~d} x \leq \sum_{i=1}^{5} I_{i} .
\end{aligned}
$$


Using Sobolev inequalities, (8) and (9), we have

$$
\begin{aligned}
& I_{1}=\int_{0}^{1} \rho u^{2} w_{x}^{2} \mathrm{~d} x \leq|\rho|_{L^{\infty}}|u|_{L^{\infty}}^{2}\left|w_{x}\right|_{L^{2}}^{2} \leq|\rho|_{L^{\infty}}\left|u_{x}\right|_{L^{2}}^{2}\left|w_{x}\right|_{L^{2}}^{2} \leq C\left|w_{x}\right|_{L^{2}}^{2} ; \\
& I_{2}=-\int_{0}^{1}(v(\rho))_{x} u w^{2} \mathrm{~d} x=\int_{0}^{1} v(\rho) u_{x} w^{2} \mathrm{~d} x+2 \int_{0}^{1} v(\rho) u w w_{x} \mathrm{~d} x \\
& \leq C|w|_{L^{\infty}}^{2}\left|u_{x}\right|_{L^{2}}+C|u|_{L^{\infty}}|w|_{L^{\infty}}\left|w_{x}\right|_{L^{2}} \leq C\left|w_{x}\right|_{L^{2}}^{2} ; \\
& I_{3}=-\int_{0}^{1}(\lambda(\rho))_{x} u w_{x}^{2} \mathrm{~d} x=-\int_{0}^{1}\left((\lambda(\rho))_{x} /(\lambda(\rho))^{2}\right) u\left(\lambda(\rho) w_{x}\right)^{2} \mathrm{~d} x \\
& =\int_{0}^{1}(1 / \lambda(\rho))_{x} u\left(\lambda(\rho) w_{x}\right)^{2} \mathrm{~d} x=-\int_{0}^{1} \lambda(\rho) u_{x}\left(w_{x}\right)^{2} \mathrm{~d} x-2 \int_{0}^{1} u w_{x}\left(\lambda(\rho) w_{x}\right)_{x} \mathrm{~d} x \\
& \leq\left|\lambda(\rho) w_{x}\right|_{L^{\infty}}\left|u_{x}\right|_{L^{2}}\left|w_{x}\right|_{L^{2}}+2 \int_{0}^{1} u w_{x}\left(\rho w_{t}+\rho u w_{x}+v(\rho) w\right) \mathrm{d} x \\
& \leq C\left|\rho w_{t}+\rho u w_{x}+v(\rho) w\right|_{L^{2}}\left|u_{x}\right|_{L^{2}}\left|w_{x}\right|_{L^{2}}+\delta\left|\sqrt{\rho} w_{t}\right|_{L^{2}}^{2} \\
& +C|\rho|_{L^{\infty}}|u|_{L^{\infty}}^{2}\left|w_{x}\right|_{L^{2}}^{2}+C|v(\rho)|_{L^{\infty}}|u|_{L^{\infty}}|w|_{L^{\infty}}\left|w_{x}\right|_{L^{2}} \\
& \leq|\rho|_{L^{\infty}}^{1 / 2}\left|\sqrt{\rho} w_{t}\right|_{L^{2}}\left|u_{x}\right|_{L^{2}}\left|w_{x}\right|_{L^{2}}+|\rho|_{L^{\infty}}|u|_{L^{\infty}}\left|w_{x}\right|_{L^{2}}^{2}+|v(\rho)|_{L^{\infty}}\left|w_{x}\right|_{L^{2}}^{2} \\
& +\delta\left|\sqrt{\rho} w_{t}\right|_{L^{2}}^{2}+C\left|w_{x}\right|_{L^{2}}^{2} \\
& \leq C\left|w_{x}\right|_{L^{2}}^{2}+2 \delta\left|\sqrt{\rho} w_{t}\right|_{L^{2}}^{2} \text {; } \\
& I_{4}=-\int_{0}^{1} v^{\prime}(\rho) \rho u_{x} w^{2} \mathrm{~d} x \leq\left|v^{\prime}(\rho)\right|_{L^{\infty}}|\rho|_{L^{\infty}}|w|_{L^{\infty}}^{2}\left|u_{x}\right|_{L^{2}} \leq C\left|w_{x}\right|_{L^{2}}^{2} ; \\
& I_{5}=-\int_{0}^{1} \lambda^{\prime}(\rho) \rho u_{x} w_{x}^{2} \mathrm{~d} x \leq-\int_{0}^{1}\left(\lambda^{\prime}(\rho) \rho / \lambda(\rho)\right) \lambda(\rho) w_{x} u_{x} w_{x} \mathrm{~d} x \\
& \leq C\left|\lambda(\rho) w_{x}\right|_{L^{\infty}}\left|u_{x}\right|_{L^{2}}\left|w_{x}\right|_{L^{2}} \leq C\left|\left(\lambda(\rho) w_{x}\right)_{x}\right|_{L^{2}}\left|u_{x}\right|_{L^{2}}\left|w_{x}\right|_{L^{2}} \\
& \leq \delta\left|\sqrt{\rho} w_{t}\right|_{L^{2}}^{2}+C\left|w_{x}\right|_{L^{2}}^{2} .
\end{aligned}
$$

Substituting the above estimates into (11), choosing $\delta=1 / 6$, and then integrating with respect to $t$ over $(0, t)$, we get

$$
\begin{aligned}
& \int_{0}^{t}\left|\sqrt{\rho} w_{t}\right|_{L^{2}}^{2} \mathrm{~d} s+|\sqrt{v(\rho)} w|_{L^{2}}+\left|\sqrt{\lambda(\rho)} w_{x}\right|_{L^{2}} \\
& \leq\left|\sqrt{v\left(\rho_{0}\right)} w_{0}\right|_{L^{2}}+\left|\sqrt{\lambda\left(\rho_{0}\right)} w_{0 x}\right|_{L^{2}}+C \int_{0}^{t}\left|w_{x}\right|_{L^{2}}^{2} \mathrm{~d} s \\
& \quad \leq C+C \int_{0}^{t}\left|\sqrt{\lambda(\rho)} w_{x}\right|_{L^{2}}^{2} \mathrm{~d} s
\end{aligned}
$$

which completes the proof of (10), according to Gronwall's inequality. 
Lemma 2.4. Under the conditions of Theorem 1.1, we have

$$
\int_{0}^{T}\left|u_{x}\right|_{L^{\infty}}^{2} \mathrm{~d} t \leq C \quad \text { and } \quad \int_{0}^{T}\left|w_{x}\right|_{L^{\infty}}^{2} \mathrm{~d} t \leq C .
$$

Proof. The first inequality has been proved in [8]; now, we consider the second inequality. By virtue of (8), then

$$
\begin{aligned}
\left|w_{x}\right|_{L^{\infty}} & \leq C\left|\lambda(\rho) w_{x}\right|_{L^{\infty}} \leq C\left|\left(\lambda(\rho) w_{x}\right)_{x}\right|_{L^{2}} \\
& \leq C\left|\rho w_{t}+\rho u w_{x}+v(\rho) w\right|_{L^{2}} \\
& \leq C|\rho|_{L^{\infty}}\left|\sqrt{\rho} w_{t}\right|_{L^{2}}+C|\rho|_{L^{\infty}}|u|_{L^{\infty}}\left|w_{x}\right|_{L^{2}}+C|w|_{L^{2}} \\
& \leq C+C\left|\sqrt{\rho} w_{t}\right|_{L^{2}} .
\end{aligned}
$$

The above inequalities together with (10) provide the proof of the second inequality.

Lemma 2.5. Under the conditions of Theorem 1.1, we have

$$
\begin{aligned}
& \sup _{0 \leq t \leq T}\left|\rho_{x}\right|_{L^{2}}^{2}+\int_{0}^{T}\left|u_{x x}\right|_{L^{2}}^{2} \mathrm{~d} t \leq C, \\
& \int_{0}^{T}\left|w_{x x}\right|_{L^{2}}^{2} \mathrm{~d} t \leq C .
\end{aligned}
$$

Proof. For the proof of (13), see [8]. From (2), (4), and (8), we have

$$
\begin{aligned}
\left|w_{x x}\right|_{L^{2}}^{2} & \leq C\left|\lambda(\rho) w_{x x}\right|_{L^{2}}^{2} \\
& \leq C\left|\lambda(\rho) w_{x x}+\lambda^{\prime}(\rho) \rho_{x} w_{x}\right|_{L^{2}}^{2}+C\left|\lambda^{\prime}(\rho) \rho_{x} w_{x}\right|_{L^{2}}^{2} \\
& \leq C\left|\left(\lambda(\rho) w_{x}\right)_{x}\right|_{L^{2}}^{2}+C\left|w_{x}\right|_{L^{\infty}}^{2}\left|\rho_{x}\right|_{L^{2}}^{2} \\
& \leq C\left|\rho w_{t}+\rho u w_{x}+v(\rho)\right|_{L^{2}}^{2}+C\left|w_{x}\right|_{L^{\infty}}^{2}\left|\rho_{x}\right|_{L^{2}} \\
& \leq C\left|\sqrt{\rho} w_{t}\right|_{L^{2}}^{2}+C|u|_{L^{\infty}}\left|w_{x}\right|_{L^{2}}^{2}+C|w|_{L^{2}}^{2}+C\left|w_{x}\right|_{L^{\infty}}^{2}\left|\rho_{x}\right|_{L^{2}}
\end{aligned}
$$

which together with (6), (7), (10), (12), and (13) furnishes the proof of (14).

\section{Proof of Theorem 1.1}

In this section, we prove the global existence of strong solutions to the problems (2)(3) by applying the a priori estimates established in the previous section. One of the main issues is the non-vanishing characteristic of the density in the approximate solutions. To this end, we modify the initial data, and choose the smooth approximate function $\left(\varrho_{0}^{\varepsilon}, u_{0}^{\varepsilon}, w_{0}^{\varepsilon}\right)$ such that

$$
\varrho_{0}^{\varepsilon} \in C^{2}[0,1], \quad 0<\varepsilon \leq \varrho_{0}^{\varepsilon} \leq 1+\left|\varrho_{0}\right|_{L^{\infty}} \quad \text { in }(0,1), \quad \varrho_{0}^{\varepsilon} \rightarrow \varrho_{0} \quad \text { in } H^{1}
$$

and

$$
\left(u_{0}^{\varepsilon}, w_{0}^{\varepsilon}\right) \rightarrow\left(u_{0}, w_{0}\right) \quad \text { in } H^{1} .
$$

Now, we consider the initial-boundary value problems (2)-(3) with the initial data $\left(\rho_{0}, u_{0}, w_{0}\right)$ replaced by $\left(\varrho_{0}^{\varepsilon}, u_{0}^{\varepsilon}, w_{0}^{\varepsilon}\right)$. By virtue of Lemmas $2.1-2.5$, we could conclude that 


$$
\sup _{0 \leq t \leq T}\left(\left|\rho^{\varepsilon}\right|_{H^{1}}+\left|\rho_{t}^{\varepsilon}\right|_{L^{2}}+\left|\left(u^{\varepsilon}, w^{\varepsilon}\right)\right|_{H^{1}}\right)+\int_{0}^{T}\left|\left(\left(\rho^{\varepsilon} u^{\varepsilon}\right)_{t},\left(\rho^{\varepsilon} u^{\varepsilon}\right) t\right)\right|_{L^{2}}^{2}+\left|\left(u_{x x^{\prime}}^{\varepsilon} w_{x x}^{\varepsilon}\right)\right|_{L^{2}} \mathrm{~d} t \leq C .
$$

We emphasize that $C$ does not depend on the parameter $\varepsilon$, i.e., the lower bound of the initial density. Then by the standard argument of compactness, we conclude from (2)-(3) that there exists a global strong solution, details of which are omitted here.

\section{Acknowledgements}

The author is indebted to the referee for giving nice suggestions which have helped them improve the presentation of this article.

\section{Author details}

${ }^{1}$ College of Mathematical Sciences, Xiamen University, Xiamen 361005, PR China ${ }^{2}$ School of Mathematics and Statistics, Shandong University at Weihai, Weihai 264209, PR China

\section{Competing interests}

The author declares that they have no competing interests.

Received: 19 March 2011 Accepted: 15 August 2011 Published: 15 August 2011

\section{References}

1. Eringen, AC: Theory of micropolar fluids. J Math Mech. 16, 1-18 (1966)

2. Mujakovic, N: One-dimensional flow of a compressible viscous micropolar fluid: a global existence theorem. Glasnic matematički. 33, 199-208 (1998)

3. Mujakovic, N: Global in time estimates for one-dimensional compressible viscous micropolar fluid model. Glasnic matematički. 40, 103-120 (2005)

4. Mujakovic, N: Non-homogeneous boundary value problem for one-dimensional compressible viscous micropolar fluid model: a global existence theorem. Math Inequalities Appl. 12(3), 651-662 (2009)

5. Liu, TP, Xin, ZP, Yang, T: Vacuum states of compressible flow. Discrete Contin Dyn Syst. 4, 1-32 (1998)

6. Chen, MT: Global strong solutions for the viscous, micropolar, compressible flow. J Part Differential Equations. 24, 158-164 (2011)

7. Amirat, Y, Hamdache, K: Weak solutions to the equations of motion for compressible magnetic fluids. J Math Pure Appl. 91, 433-467 (2009). doi:10.1016/j.matpur.2009.01.015

8. Wen, HY, Yao, L: Global existence of strong solutions of the Navier-Stokes equations for isentropic compressible fluids with density-dependent viscosity. J Math Anal Appl. 349, 503-515 (2009). doi:10.1016/j.jmaa.2008.09.025

9. Hoff, D: Global solutions of the equations of one-dimensional, compressible flow with large data and forces, and with differing end states. Z Angew Math Phys. 49, 774-785 (1998). doi:10.1007/PL00001488

10. Guo, ZH, Zhu, CJ: Global weak solutions and asymptotic behavior to 1D compressible Navier-Stokes equations with density-dependent viscosity and vacuum. J Differential Equations. 248, 2768-2799 (2010). doi:10.1016/j.jde.2010.03.005

11. Jiang, S, Xin, ZP, Zhang, P: Global weak solutions to 1D compressible isentropic Navier-Stokes equations with densitydependent viscosity. Methods Appl Anal. 12, 239-251 (2005)

doi:10.1186/1687-2770-2011-13

Cite this article as: Chen: Global existence of strong solutions to the micro-polar, compressible flow with density-dependent viscosities. Boundary Value Problems 2011 2011:13.

\section{Submit your manuscript to a SpringerOpen ${ }^{\circ}$ journal and benefit from:}

Convenient online submission

- Rigorous peer review

- Immediate publication on acceptance

- Open access: articles freely available online

- High visibility within the field

- Retaining the copyright to your article

Submit your next manuscript at $\mathbf{s p r i n g e r o p e n . c o m ~}$ 